\title{
A Rapid and Sensitive Bio Analytical RP-HPLC Method for Detection of Docetaxel: Development and Validation
}

\author{
Prachi Balaram Kharkar\#, Swapnil Sunil Talkar\#, Vandana Bharat Patravale* \\ Department of Pharmaceutical Sciences and Technology, Institute of Chemical Technology, Nathalal Parekh Marg, Matunga, Mumbai, \\ INDIA.
}

\begin{abstract}
Objective: A rapid and selective high-performance liquid chromatographic (HPLC) method has been developed and validated for Docetaxel anhydrous (DTX) using Ketoconanzole (KCZ) as an internal standard in biological fluids. Methods: The analyte was extracted from human plasma by liquid-liquid extraction method using acetonitrile. The analysis was carried out on Licrosphere IV, $\mathrm{C}_{8}$ column (LC-GC Chromatography Solutions Pvt. Ltd, Mumbai, and India) $(4.6 \times 250 \mathrm{~mm})$ with isocratic elution using a mobile phase composition of $0.2 \%$ triethylamine $(\mathrm{pH} 6.4$ with orthophosphoric acid) $(45 \%)$ and Acetonitrile $(55 \%)$. The flow rate was $1.5 \mathrm{ml} / \mathrm{min}$ with absorbance wavelength of 230 $\mathrm{nm}$. Results: The developed method was found to be linear over the range of 100$2500 \mathrm{ng} / \mathrm{mL}$ with correlation coefficients of about 0.999. The limit of quantification for docetaxel was found to be $100 \mathrm{ng} / \mathrm{mL}$. The limit of detection was found to be $50 \mathrm{ng} /$ $\mathrm{mL}$. The intra- and inter-day precision was determined to be $<5 \%$ and the accuracy was $99-105 \%$. The overall recovery for docetaxel was about $89.0 \%-91.0 \%$. The total analysis time was only $10.0 \mathrm{~min}$. The method was successfully applied for determination of DTX in rat plasma, to establish the pharmacokinetic profile of a marketed formulation. Conclusion: A simple, sensitive, rapid, accurate and prudent RP-HPLC method was developed, validated and applied for the Docetaxel anhydrous.
\end{abstract}

Key words: Docetaxel anhydrous, Ketoconazole, Bio-analytical method, HPLC, Pharmacokinetic study.

\section{INTRODUCTION}

Docetaxel (DTX), an anti-neoplastic chemotherapeutic drug (family taxoid) is obtained synthetically from 10-deacetyl baccatin III, extracted from the biomass of the Yew plant. It has a high binding affinity for microtubules resulting in their depolymerization; especially at the plus end side which leads to the inhibition of mitotic cell division between metaphase and anaphase. DTX is approved by the FDA for the treatment of metastasis prostate cancer, breast cancer, non-small cell lung cancer, advanced stomach cancer, head and neck cancer; while also being investigated to treat ovarian, bladder, pancreatic and small cell lung cancer, melanoma and soft tissue sarcoma.
Bio analytical methods are used for the quantitative determination of analyte and their metabolites in biological matrix like saliva, plasma, urine, serum etc. This quantification plays important role in evaluation and interpretation of bioequivalence, bioavailability, pharmacological and pharmacokinetic data. ${ }^{1}$ Validation for bio analytical method should include demonstration of calibration curve, accuracy, recovery, precision, sensitivity, reproducibility, and stability of analyte in spiked samples. It is a tool to ensure reproducibility and robustness of the given method for quantitative measurement of an analyte in a given biological sample., ${ }^{2,3}$ There are number of thera-
Submission Date: 22-08-2017; Revision Date: 17-09-2017; Accepted Date: 25-10-2017.

DOI: 10.5530/ijper.51.4s.105 Correspondence: Dr. Vandana B Patravale, Department of Pharmaceutical Sciences and Technology, Institute of Chemical Technology, Matunga East, Mumbai- 400019, INDIA. Phone no: 91-22-3361 2217 E-mail:vb.patravale@ ictmumbai.edu.in

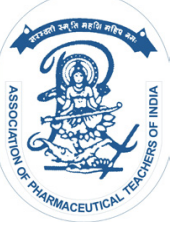

www.ijper.org 
peutic agents such as synthetic drugs, recombinant proteins, vaccines etc. involved in chemotherapy. Owing to this, it is necessary to develop and validate a bio analytical method for the quantification of these therapeutics in biological samples. ${ }^{4}$

Several chromatographic methods are available in literature for the plasma detection of docetaxel. Most of the methods report the use of high performance liquid chromatography along with UV, Fluorescence, Mass spectroscopy, etc. as the detection technique. However, most of these methods employ paclitaxel (docetaxel analogue) as the internal standard which is as expensive as docetaxel and difficult to procure. Moreover, most of the reported methods are very time-consuming which further increases the cost of analysis.., 6

The current research work addresses this issue by focusing on development of a simple, rapid and robust method for the determination of DTX in plasma, using Ketoconazole as an internal standard. Ketoconazole (KCZ) is BCS Class II molecule and has a $\lambda_{\text {max }}$ of $230 \mathrm{~nm}$, similar to that of DTX. Chromatographic run time was minimized to provide fast quantitative detection. Furthermore, the method was validated based on parameters like accuracy, precision, specificity, robustness and was also utilized to evaluate the pharmacokinetic profile of DTX.

\section{MATERIALS AND METHODS}

DTX was obtained as a gift sample from Dr. Reddy's Laboratory, Hyderabad, India. Ketoconazole gift sample was procured from Aarti chemicals Pvt. Ltd., Dombivali, India. HPLC grade acetonitrile and orthophosphoric acid and triethylamine were purchased from Merck Millipore, Mumbai, India and S.D. Fine Chemicals, Mumbai, India respectively. Further, $0.45 \mu \mathrm{m}$ membranes were purchased from Pall Life Sciences. Ultra-pure water using Milli Q Plus purification system (Millipore, Bedford, MA, USA) was used for the preparation of mobile phase. All other chemicals used were of analytical grade unless otherwise indicated.

\section{Instrumentation}

\section{HPLC}

The HPLC system consisted of Agilent 1100 modules (G1310A Isocratic Pump with solvent container, G1314A VW Detector with standard flow cell and G1328A Manual Injector). The output signal was monitored and processed using an Agilent single G2220AA 2D-Value Solution ChemStation.

\section{Chromatographic conditions}

HPLC method described by Venishetty et al. is modified in this research work, for bio analytical determination of docetaxel to reduce the run time. ${ }^{7}$ Chromatographic separation was achieved on a $5 \mu \mathrm{m}$, Licrosphere IV, $\mathrm{C}_{8}$ column $(4.6 \mathrm{~mm} \times 250 \mathrm{~mm})$ procured from LC-GC Chromatography Solutions Pvt. Ltd, Mumbai, India. Different mobile phase compositions were tried to achieve optimum detection of analyte. The optimum results were obtained at mobile phase composition of $0.2 \%$ triethylamine ( $\mathrm{pH} 6.4$ with orthophosphoric acid) $(45 \%)$ and Acetonitrile (55\%). Prior to use, the mobile phase system was filtered through a $0.45 \mu \mathrm{m}$ filter membrane and degassed. The mobile phase was pumped through the column at a flow rate of $1.5 \mathrm{~mL} / \mathrm{min}$. Ketoconazole was used as an internal standard (IS). The injection volume was $100 \mu \mathrm{L}$. The mobile phase system was used for the dilution of the standard and sample solutions. The analyte was analysed at a single wavelength of $230 \mathrm{~nm}$.

\section{Experimental}

\section{Preparation of stock solutions}

Primary stock solutions of docetaxel and the internal standard (IS) were prepared separately by dissolving $2 \mathrm{mg}$ accurately weighed standard compounds in $1 \mathrm{~mL}$ ethanol to produce a concentration of $2000 \mathrm{ppm}$, and were stored at $2-8^{\circ} \mathrm{C}$. A series of working standard solutions of each analyte at appropriate concentrations were obtained by mixing and further diluting of the stock solution with ethanol. A working standard solution of the IS $\left(2 \mathrm{mg} \mathrm{mL}^{-1}\right)$ was prepared by diluting IS stock solution with methanol. These diluted working standard solutions were used to prepare the calibration curve and quality control (QC) samples in human plasma.

\section{Calibration standards and QC samples}

Blank human plasma was screened prior to spiking to ensure it was free of endogenous interference at the retention times of docetaxel and ketoconazole. Standard calibration samples were prepared by spiking the blank human plasma with working solutions of each analyte, to yield six different concentrations over a range of $100-2500$ ngmL $^{1}$ for docetaxel (i.e. 100, 500, 1000,

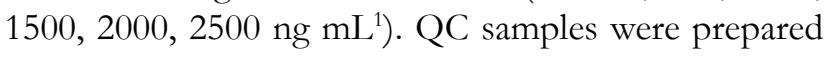
by adding appropriate volumes of QC working solutions (from a separate weighing to that for the calibration standard) to blank human plasma, to yield the low, medium and high concentrations (500, 1500, $2500 \mathrm{ng} \mathrm{mL}^{1}$ for docetaxel). The concentration of the IS was $500 \mathrm{ng} \mathrm{mL}^{1}$ in all samples. 


\section{Sample preparation}

QC samples, calibration standards, and human plasma samples were extracted by the liquid-liquid extraction (LLE) technique with acetonitrile. A volume of $100 \mu \mathrm{L}$ of drug-free human plasma was added to a disposable Eppendorf tube, followed by spiking with $100 \mu \mathrm{L}$ of the standard working solution and $30 \mu \mathrm{L}$ of IS working solution, respectively. The mixture was vortexes for 30s using a vortex mixer. Then, a single-step LLE was adopted to extract all the analytes from the human plasma. For this purpose, $770 \mu \mathrm{L}$ of acetonitrile was added to each tube followed by vortexing for $2 \mathrm{~min}$. The well-vortexes solutions were then centrifuged at 10000 $\mathrm{rpm}$ for $15 \mathrm{~min}$. The supernatant was filtered through $0.45 \mu \mathrm{m}$ syringe driven membrane filter unit (Pall India Pvt. Ltd., Ahmedabad, India) and was used to prepare calibration standards.

\section{Method validation}

The method was validated according to the International Conference on Harmonization (ICH) bio analytical method validation guidelines Q2 (R1) with respect to specificity, linearity, sensitivity, accuracy, and precision. The matrix effects as well as the stability of analytes in plasma were also determined.

\section{Specificity}

The specificity of the method was investigated by analysing six different batches of drug-free human plasma (without IS or analytes) for the exclusion of any endogenous co-eluting interferences at the peak region of each analyte and the IS.

\section{Linearity and sensitivity}

The calibration standards were prepared and assayed in triplicate on three different days to demonstrate the linearity of this method. The lower limit of quantification (LLOQ) was defined as the lowest concentration at which the signal to noise $(\mathrm{S} / \mathrm{N})$ ratio was larger than 10 and both the precision and accuracy were less than or equal to $20 \%$ by analysing the triplicates of samples spiked with each analyte.

\section{Precision and Accuracy}

The precision and accuracy of the entire method were assessed at three QC concentration levels (i.e. 500, 1500, 2500 ngmL ${ }^{1}$ for docetaxel), each extracted and analysed in triplicates on the same day (intra-day precision and accuracy) and on three different days within 1 week (inter-day precision and accuracy). Precision was expressed as the relative standard deviation (RSD) and accuracy was calculated as the relative difference between calculated and nominal concentration of the
QC samples (bias\%).The intra- and inter-day precision and bias were set at $<15 \%$, except that at LLOQ, where it was set at $<20 \%$.

\section{Recovery}

The extraction recovery (absolute recovery) was determined by measuring an extracted sample against un-extracted standards and expressed as the ratio of the peak responses. For docetaxel, the recovery experiments were performed with three QC concentrations (low, medium and high QC concentrations), with triplicate determinations at each concentration.

\section{Matrix effect}

The matrix effect on the ionization efficiency of each analyte was evaluated by comparing the peak response of analytes dissolved in blank sample extract (i.e., the final solution obtained from blank plasma after extraction and reconstitution) with those for analytes dissolved to the same concentrations in acetonitrile. The experiment was performed in triplicate for each QC concentration. If the peak area ratios for the plasma extracts vs. clean acetonitrile solutions were $<85 \%$ or $>115 \%$, a matrix effect was implied.

\section{Stability}

The stability testing was determined in four ways: (1) for storage stability, the QC samples were prepared and stored at $-20^{\circ} \mathrm{C}$ for 2 weeks. All samples were subsequently thawed and analysed together with calibration samples which were freshly prepared. (2) For freeze/ thaw stability testing, the QC samples were determined after three freeze $\left(-20^{\circ} \mathrm{C}\right.$, storage temperature)/thaw (ambient temperature) cycles and analysed with the freshly prepared calibration samples. (3) To investigate the stability during sample processing, the QC samples were left at $25 \pm 2^{\circ} \mathrm{C}$ for $6 \mathrm{~h}$, the average time required for sample preparation, and then analysed with the freshly prepared calibration samples. For each of the above stability tests, the experiments were performed at three QC concentrations (low, medium and high), with triplicate determinations for each concentration. The obtained results were compared with the nominal concentration of the analytes. A compound was considered unsTable if the calculated concentration was less than the nominal concentration by more than $15 \%$.

\section{Application of method}

The animal experiments were executed in compliance with the guidelines of the Committee for the Purpose of Control and Supervision of Experimental Animals (CPCSEA), India. The protocol was approved by Institutional Animal Ethics Committee (IAEC).Six healthy 
females Sprague-Dawley rats (200-250 gms), procured from National Institute of Biosciences, Pune were used for study. The rats were allowed to adapt to the housing environment for at least 1 week prior to study. The rats were intravenously (i.v.) administered with marketed formulation Daxotel ${ }^{\circledR}$ (manufactured by Fresenius Kabi Oncology Ltd.) equivalent to $2 \mathrm{mg} / \mathrm{kg}$ docetaxel via tail vein injection using $1 \mathrm{~mL}$ syringe. Blood samples (approx. $0.5 \mathrm{~mL}$ ) were collected from retro orbital plexuses at time intervals of $0.5,1,2,4,6,8,12,24$ and 48 hrs. Plasma samples collected were centrifuged at $5000 \mathrm{rpm}$ for $15 \mathrm{~min}$, to separate $200 \mu \mathrm{L}$ plasma, which was kept at $-20^{\circ} \mathrm{C}$ until analysis. These samples were treated using the above mentioned bio analytical method.

\section{RESULTS AND DISCUSSION}

\section{Selectivity and Specificity}

All analytes eluted rapidly with good resolution within $10 \mathrm{~min}$. The plasma matrix components were not interfering with the analyte peaks Figure 1. Docetaxel eluted at $4.9 \mathrm{~min}$ and the IS eluted at $7.9 \mathrm{~min}$. No interfering endogenous peaks were detected thus indicating that the method possesses high specificity from endogenous substances and other associated agents. Peak shape and retention time were found to be same as that of pure standards.

\section{Linearity}

The developed method was found to be linear in the range of $100-2500 \mathrm{ng} \mathrm{mL}^{1}$ for the analyte. Good linearity with a correlation coefficient $r^{2}$ exceeding 0.999 was observed for analyte. The representative regression equations were $\mathrm{y}=0.001 \mathrm{x}+0.021$, where $\mathrm{y}$ indicates the ratio of analyte to internal standard and $\mathrm{x}$ indicates the plasma concentration. The lower limits of quantification (LLOQs) under the optimized conditions were $100 \mathrm{ng} \mathrm{mL}{ }^{1}$ for docetaxel, which was determined from visual method of detection.

\section{Precision and Accuracy}

For evaluation of precision and accuracy, three different QC samples i.e. lower, medium and higher in triplicate were analysed. Results are depicted in Table 1.

The intra-day precision (\% relative standard deviation ( $\% \mathrm{RSD}))$ of the assay was less than $4 \%$ for each of the three concentrations of the QC samples; assay accuracy was in the range $99-101 \%$. The inter-day precision ( $\%$ RSD) of the assay was less than 3\% for all the QC samples, and assay accuracy was in the range $100-103 \%$. At these concentrations, the intra- and inter-day \%RSD

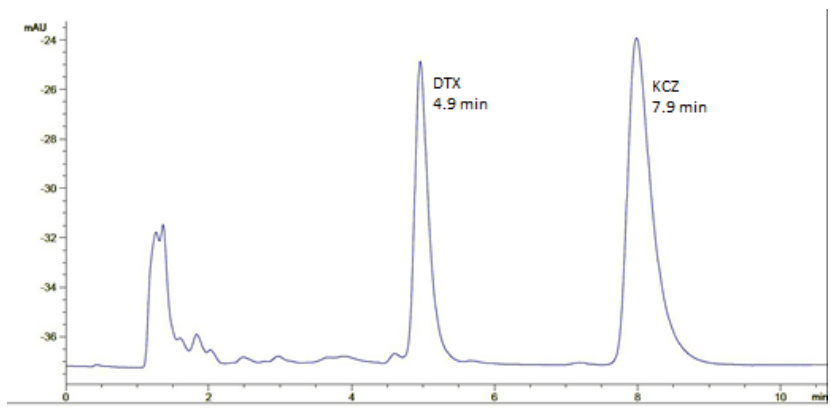

Figure 1: Representative Chromatogram of DTX and IS (KCZ).

was determined to be $<5 \%$, and the accuracy was 99-105\%. These outcomes imply that the developed method is accurate, precise and reproducible for detecting docetaxel over its therapeutic range.

\section{Recovery and Matrix effect}

The extraction recoveries from the human plasma were determined at the concentrations of 500, 1500, $2500 \mathrm{ng} \mathrm{mL}{ }^{1}$ for docetaxel in triplicate. The one-step LLE was proved to be simple, rapid and successful with an average recovery ratio of about $89-91 \%$ for docetaxel at the tested concentrations Table 2 Recovery of docetaxel was found to be less as the drug is highly protein bound $(\sim 97 \%){ }^{8,9}$ Hence the $\%$ RSD for recoveries was found to be all below $8 \%$. The possibility of matrix effect caused by ionization competition between the analytes and the endogenous co-eluents was evaluated at three QC concentrations in triplicate. The results of matrix effect were acquired from comparing the peak responses of the post-extraction spiked samples with those of the standard sample solution. The matrix effect in this method was suggested to be negligible.

\section{Stability}

No significant degradation (the losses were within 4.0\%) of docetaxel was observed during all of the sample storage, preparation and analysis periods. Results of the stability evaluation are shown in Table 3.

\section{Application of method}

The method has been successfully applied to the analysis of samples from a pharmacokinetic study in SD rats. Figure 2 depicts a pharmacokinetic profile of docetaxel in rat plasma after i.v. administration of marketed formulation to six rats. The clearance time was found to be $7.6474 \pm 0.0631$ and half-life $\left(t_{1 / 2}\right)$ was $0.704 \pm 0.007 \mathrm{hr}$. The parameters are in accordance with those reported previously. 


\section{Table 1: Intra and Inter-day precision and accuracy for the determination of} DTX in human plasma.

\begin{tabular}{|c|c|c|c|}
\hline $\begin{array}{c}\text { Concentration } \\
\text { (spiked)ng/mL }\end{array}$ & $\begin{array}{c}\text { Concentration } \\
\text { (measured) } \\
\text { ng/mL }\end{array}$ & $\begin{array}{c}\text { Precision } \\
\text { (\% RSD) }\end{array}$ & $\begin{array}{c}\text { Accuracy } \\
\text { (\% } \pm \text { SD) }\end{array}$ \\
\hline Intraday & 104.56 & 3.15 & $104.56 \pm 2.83$ \\
\hline 100 & 504.14 & 0.58 & $100.08 \pm 2.92$ \\
\hline 500 & 1506.21 & 0.29 & $100.41 \pm 4.39$ \\
\hline 1500 & 2498.74 & 0.04 & $99.94 \pm 0.890$ \\
\hline 2500 & 102.98 & 2.08 & $102.98 \pm 2.107$ \\
\hline Interday & 507.29 & 1.02 & $101.45 \pm 5.15$ \\
\hline 100 & 1504.59 & 0.22 & $100.3 \pm 3.24$ \\
\hline 500 & 2503.47 & 0.1 & $100.13 \pm 2.45$ \\
\hline 1500 & & & \\
\hline
\end{tabular}

\begin{tabular}{|c|c|c|c|}
\hline \multicolumn{4}{|c|}{ Table 2: Extraction efficiency (\%) of DTX in human plasma. } \\
\hline $\begin{array}{c}\text { Concentration } \\
\text { spiked }\end{array}$ & $\begin{array}{c}\text { Concentration } \\
\text { measured }\end{array}$ & \% Recovery & \% RSD \\
\hline DTX & & & 6.49 \\
\hline 500 & 452.82 & 90.56 & 7.14 \\
\hline 1500 & 1348.35 & 89.89 & 7.06 \\
\hline 2500 & 2231.85 & 89.27 & \\
\hline KCZ (IS) & & & 0.37 \\
\hline 500 & 502.68 & 100.53 & \\
\hline
\end{tabular}

\begin{tabular}{|c|c|c|c|c|}
\hline \multicolumn{5}{|c|}{ Table 3: Stability studies for DTX. } \\
\hline Concentration (ng/mL) & $\begin{array}{c}\text { Freeze thaw } \\
\text { stability }\end{array}$ & $\begin{array}{c}\text { Short term } \\
\text { stability }\end{array}$ & $\begin{array}{c}\text { Post preparative } \\
\text { Stability }\end{array}$ & $\begin{array}{c}\text { Long term } \\
\text { Stability }\end{array}$ \\
\hline \multicolumn{5}{|c|}{$\%$ Recoveries (mean \pm SD) } \\
\hline 500 & $97.33 \pm 9.41$ & $100.58 \pm 2.07$ & $100.37 \pm 1.32$ & $100.73 \pm 2.58$ \\
\hline 1500 & $99.33 \pm 7.10$ & $100.06 \pm 0.72$ & $100.14 \pm 1.5$ & $100.04 \pm 0.47$ \\
\hline 2500 & $99.62 \pm 6.65$ & $100.02 \pm 0.48$ & $100.11 \pm 2.1$ & $100.06 \pm 1.18$ \\
\hline
\end{tabular}

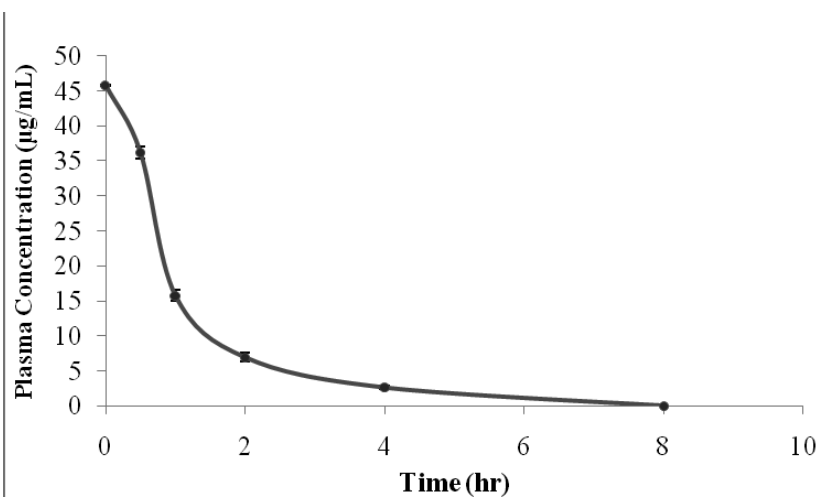

Figure 2: Plasma concentration versus time profile of docetaxel after i.v. administration.

\section{CONCLUSION}

The bio analytical method for docetaxel was developed using ketoconazole as internal standard, which offers substantial benefits in terms of sensitivity, short analysis time and selectivity. The method was validated as per ICH guidelines and was found to comply with all the parameters of validation within the said limits. Developed method shows acceptable values of recovery and can be used to estimate docetaxel from the biological matrix like saliva, plasma, urine, serum etc. From the results obtained we conclude that the method was found to be accurate and precise and was successfully applied to study the pharmacokinetics profile of a marketed formulation. 


\section{ACKNOWLEDGEMENT}

Authors would like thank University Grants Commission - Basic Science Research (UGC-BSR) and Joint Council of Scientific and Industrial Research - University Grants Commission (CSIR-UGC), Government of India for providing research fellowship.

\section{CONFLICT OF INTEREST}

Authors declare no conflict of interest.

\section{ABBREVIATIONS USED}

HPLC: High Performance Liquid Chromatography; DTX: Docetaxel anhydrous; KCZ: Ketoconazole; IS: Internal standard; QC: Quality control; LLE: Liquid liquid extraction; RSD: Relative standard deviation.

\section{REFERENCES}

1. Bressolle F, Bromet-Petit M, Audran M. Validation of liquid chromatographic and gas chromatographic methods Applications to pharmacokinetics. Journal of Chromatography B: Biomedical Sciences and Applications. 1996;686(1):310.

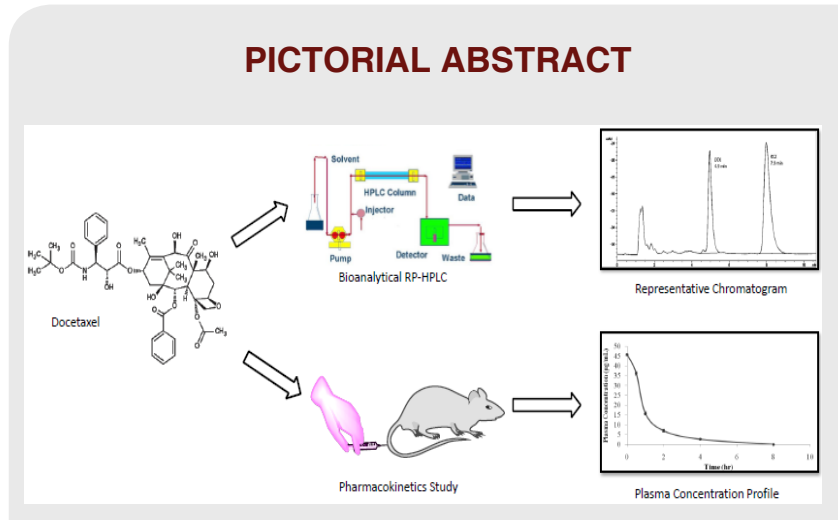

\section{About Authors}

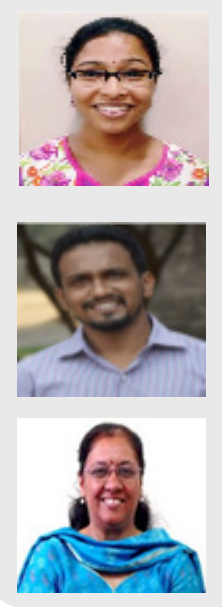

Ms. Prachi Kharkar: Is an UGC-BSR senior research fellow of Department of Pharmaceutical Sciences and Technology at Institute of Chemical Technology, Mumbai, India.

Mr. Swapnil Talkar: Is a CSIR-UGC senior research fellow of Department of Pharmaceutical Sciences and Technology at Institute of Chemical Technology, Mumbai, India.

Dr. Vandana Patravale: Is a Professor of Pharmaceutics of Department of Pharmaceutical Sciences and Technology at Institute of Chemical Technology, Mumbai, India.

Cite this article: Kharkar PB, Talkar SS, Patravale VB. A Rapid and Sensitive Bio Analytical RP-HPLC Method for Detection of Docetaxel: Development and Validation. Indian J of Pharmaceutical Education and Research. 2017;51(4S):S729-S34.
2. Reid E, Wilson ID. Analysis for drugs and metabolites, including anti-infective agents. In International Bioanalytical Forum 1989: Guilford, England;Royal Society of Chemistry. 1990.

3. US Food and Drug Administration. Guidance for Industry on Bioanalytical Method Validation Center for Drug Evaluation and Research, US Food and Drug Administration: Rockville, MD, 2001. www.fda.gov/downloads/Drugs/ Guidances/ucm070107.pdf (date of accession: January, 12 2017)

4. Kirthi A, Shanmugam R, Prathyusha MS,Basha DJ. A review on bioanalytical method development and validation by RP-HPLC. Journal of Global Trends in Pharmaceutical Sciences. 2014;5(4):2265-71.

5. Garg MB, Ackland SP. Simple and sensitive high-performance liquid chromatography method for the determination of docetaxel in human plasma or urine. Journal of Chromatography B: Biomedical Sciences and Applications. 2000;748(2):383-8.

6. Kuppens IELM, Van Maanen MJ, Rosing H, Schellens JHM, Beijnen JH. Quantitative analysis of docetaxel in human plasma using liquid chromatography coupled with tandem mass spectrometry. Biomedical Chromatography. 2005;19(5):355-61.

7. Venishetty VK, Parikh N, Sistla R, Ahmed FJ, Diwan PV. Application of validated RP-HPLC method for simultaneous determination of docetaxel and ketoconazole in solid lipid nanoparticles. Journal of Chromatographic Science. 2011;49(2):136-41.

8. Urien S, Barré J, Morin C, PaccalyUrien, S, Morin C, Paccaly A, et al. Docetaxel serum protein binding with high affinity to alpha 1-acid glycoprotein. Investigational New Drugs. 1996;14(2):147-51.

9. Sylvia S, Ng SS, Figure, WD, Sparreboom A. Taxane-Mediated Antiangiogenesis in vitro. Cancer Research. 2004;64(3):821-4.

- A rapid, specific, sensitive and cost effective bioanalytical (RP- HPLC) method for estimation of docetaxel from plasma was developed to obviate the use of paclitaxel as an internal standard. The developed method utilizes Licrosphere IV, $\mathrm{C}_{8}$ column $(4.6 \times 250 \mathrm{~mm})$ as a stationary phase and $0.2 \%$ triethylamine $(\mathrm{pH}$ 6.4 with orthophosphoric acid) and Acetonitrile in the ratio of $45: 55$ as a mobile phase and ketoconazole as an internal standard. The run time of method was about 10 min with good resolution between plasma peaks, analyte and internal standard. The developed method was validated as per ICH Q2R1 for specificity, linearity, sensitivity, precision, accuracy, recovery and stability. The developed method was demonstrated by in vivo pharmacokinetic studies in Sprague dawley rats using marketed formulation Daxotel ${ }^{\circledR}$ (manufactured by Fresenius Kabi Oncology Ltd.). All values for precision, accuracy and recovery are found to be in range as per $\mathrm{ICH}$. The pharmacokinetic profile exhibits that the method is suitable for intended use. 